\section{OPEN ACCESS}

Edited by:

Trygue Tollefsbol,

University of Alabama at Birmingham,

United States

Reviewed by:

Myong-Hee Sung,

National Institutes of Health (NIH),

United States

Dag H. Yasui,

University of California, Davis,

United States

*Correspondence.

Chuanyu Liu

liuchuanyu@genomics.cn

Longqi Liu

liulongqi@genomics.cn

tThese authors have contributed equally to this work

Specialty section:

This article was submitted to

Epigenomics and Epigenetics,

a section of the journal

Frontiers in Genetics

Received: 10 January 2021

Accepted: 01 April 2021

Published: 24 May 2021

Citation:

Yuan Y, Deng Q, Wei X, Liu Y, Lan Q, Jiang Y, Yu Y, Guo $P, X u J, Y u C$,

Han L, Cheng M, WU P, Zhang $X$,

Lai Y, Volpe G, Esteban MA, Yang $H$, Liu C and Liu L (2021) The Chromatin

Accessibility Landscape of Adult Rat.

Front. Genet. 12:651604.

doi: $10.3389 /$ fgene.2021.651604

\title{
The Chromatin Accessibility Landscape of Adult Rat
}

\begin{abstract}
Yue Yuan ${ }^{1,2 \dagger}$, Qiuting Deng ${ }^{1,2 \dagger}$, Xiaoyu Wei ${ }^{1,2+}$, Yang Liu ${ }^{1,2}$, Qing Lan ${ }^{2}$, Yu Jiang ${ }^{3}$, Yeya Yu ${ }^{2,4}$, Pengcheng Guo ${ }^{5}$, Jiangshan $X u^{1,2}$, Cong $\mathrm{Yu}^{2}$, Lei Han ${ }^{2}$, Mengnan Cheng ${ }^{1,2}$, Peiying $W u^{2}$, Xiao Zhang ${ }^{5}$, Yiwei Lai ${ }^{6}$, Giacomo Volpe ${ }^{6}$, Miguel A. Esteban ${ }^{2,3,5,6,7}$, Huanming Yang ${ }^{2,8,9}$, Chuanyu Liu $^{2,10 *}$ and Longqi Liu ${ }^{2,10 *}$
\end{abstract}

${ }^{1}$ BGI Education Center, University of Chinese Academy of Sciences, Shenzhen, China, ${ }^{2}$ BGI-Shenzhen, Shenzhen, China, ${ }^{3}$ First Hospital, Jilin University, Changchun, China, ${ }^{4}$ BGl College, Zhengzhou University, Zhengzhou, China, ${ }^{5}$ College of Veterinary Medicine, Jilin University, Changchun, China, ${ }^{6}$ Laboratory of Integrative Biology, Guangzhou Institutes of Biomedicine and Health, Chinese Academy of Sciences, Guangzhou, China, ${ }^{7}$ Bioland Laboratory (Guangzhou Regenerative Medicine and Health Guangdong Laboratory), Guangzhou, China, ${ }^{8}$ James D. Watson Institute of Genome Sciences, Hangzhou, China, ${ }^{9}$ Guangdong Provincial Academician Workstation of BGl Synthetic Genomics, BGI-Shenzhen, Shenzhen, China, ${ }^{10}$ Shenzhen Bay Laboratory, Shenzhen, China

Keywords: rat, body organs, brain regions, chromatin accessibility, transcription factors

\section{INTRODUCTION}

The use of animal models has greatly facilitated biomedical discoveries to better understand human physiology and diseases (Baldini, 2010). For example, mice are widely used in biomedical research with advantages in fast breeding, easy feeding, and genetic engineering (Gurumurthy et al., 2020). Multi-omics datasets in mouse models [e.g., RNA-seq (Koh et al., 2016; Söllner et al., 2017; Han et al., 2018), ChIP-seq (Dahl et al., 2016; Liu et al., 2016; Zhang et al., 2016), and ATAC-seq (Assay for Transposase-Accessible Chromatin coupled with next-generation sequencing) (Wu et al., 2016; Cusanovich et al., 2018a; Liu et al., 2019)] are largely accumulating, at either tissue or single-cell levels, in both developmental (Dahl et al., 2016; Liu et al., 2016; Wu et al., 2016; Zhang et al., 2016) and pathological (Park et al., 2018) studies, further advancing our understanding of complex diseases. In contrast, while the Norway rat allows more precise modeling of complex human disorders [such as cardiovascular and psychiatric disease (Abbott, 2009; Jacob, 2010)], very few multi-omics studies have been performed on this model since the completion of the full genome sequencing of Brown Norway (BN) rat strain in 2004 (Gibbs and Pachter, 2004). In 2017, Söllner et al. (2017) were the first to conduct a comprehensive tissue-level transcriptome study on 13 tissues of rat and mouse tissues and found that, while the majority of genes are highly conserved, there are also few hundreds of genes that displayed opposite expression patterns in rats and mice. Hence, additional transcriptome and chromatin openness profilings would be of great importance to further unravel regulatory complexities of the rat genome.

In neuroscience, social behaviors such as grooming, sniffing, and chasing and their relationships with disease phenotypes have been assessed in great details in rat models (Meaney and Stewart, 1981; Schweinfurth, 2020). For example, a recent study has focused on both proteomic and transcriptomic profiling of the rat amygdala following a social play experiment and reported that the GABAergic, glutamatergic, and G-protein-coupled receptor signaling can be altered by social contexts (Alugubelly et al., 2019). By using rat as a model, future studies along this line would further illustrate mechanistic relationship between brain regions and social play behaviors.

Comprehensive omics datasets have paved the way for important discoveries in physiology, diseases, social behaviors, and many other areas. In this regard, previous studies have generated a collection of omics references (including genome, transcriptome, epigenome, and proteome) for model organisms such as human (Collins et al., 2003; Cao et al., 2020; Domcke et al., 2020), 
mouse (Cusanovich et al., 2018a; Han et al., 2018), worm (Gerstein et al., 2010; Li et al., 2014; Daugherty et al., 2017), and fly (Adams et al., 2000; Graveley et al., 2011; Cusanovich et al., 2018b). However, rat multi-omics data resources are still lacking. In this study, we comprehensively profiled the chromatin accessibility of 10 body organs (pancreas, adrenal gland, spleen, ovary, heart, ileum, lung, kidney, liver, thymus) and 12 different brain regions in female and male rats (somatosensory cortex, motor cortex, primary visual cortex, auditory cortex, prefrontal cortex, thalamus, cerebellum, striatum, hypothalamus, amygdala, hippocampus, olfactory bulb), by applying ATACseq (Buenrostro et al., 2013, 2015; Corces et al., 2017). This approach led to the identification of 397,691 chromatin accessible elements, with 34,219 body organ-specific peaks and 38,502 brain-specific peaks that were further characterized as tissuespecific regulatory elements. Interestingly, many of the enriched tissue-specific transcription factors were validated by previous studies (Liu et al., 2019). Here we provide a comprehensive rat tissue-specific chromatin accessibility landscape that would serve as an invaluable resource for future rat-related studies.

\section{MATERIALS AND METHODS}

\section{Sample Collection}

Sprague-Dawley female and male rats, $\sim 7-8$ months old, were obtained from Jiangsu ALF Biotechnology Co., Ltd. (http:// jsalfei.com) and used in the study. Experimental protocols related to the use of laboratory animals were approved by the Institutional Review Board on Ethics Committee of BGI (permit no. BGI-IRB A20020). Briefly, two female rats were sacrificed by carbon dioxide asphyxiation, and tissues were harvested and quickly frozen in liquid nitrogen and stored in liquid nitrogen, which include body organ tissues (e.g., pancreas, adrenal gland, spleen, ovary, heart, ileum, lung, kidney, liver, thymus) and brain regions (e.g., somatosensory cortex, motor cortex, primary visual cortex, auditory cortex, prefrontal cortex, thalamus, cerebellum, striatum, hypothalamus, amygdala, hippocampus, olfactory bulb). To assess gender-related variation, two male rats were also sacrificed, and the following organs were collected: pancreas, spleen, heart, lung, kidney, liver, thymus, cerebellum, epididymis, spermaduct, testis, hypothalamus, hippocampus, and olfactory bulb (Supplementary Table 1).

\section{Nuclei Isolation From Frozen Tissues}

Nuclei were isolated from flash frozen rat tissues according to a standard nuclei extraction method (Corces et al., 2017). Briefly, shredded tissue sample was transferred to a prechilled $2 \mathrm{~mL}$ tissue Dounce homogenizer (Sigma, D8938-1SET) containing $2 \mathrm{~mL}$ of cold homogenization buffer [ $10 \mathrm{mM}$ Tris-HCl, pH 8.0 (Sigma, T2694-1L), $25 \mathrm{mM} \mathrm{KCl}$ (Thermo, AM9640G), $5 \mathrm{mM} \mathrm{\textrm {MgCl } _ { 2 }}$ (Ambion, AM9530G), $250 \mathrm{mM}$ sucrose (BBI, SB0498), 0.1\% NP40 (Roche, 11332473001), 0.1\% Tween-20 (Sigma, P9416), $0.01 \%$ digitonin (Sigma, D141-100MG), $1 \times$ protease inhibitor cocktail (Roche, 4693116001), and 0.1 mM DTT (Sigma, 646563) in nuclease-free water (Ambion, AM9932)]. At this stage, samples were incubated on ice for 5-10 min. Tissues were then homogenized with 10-15 strokes using pestle A (loose), followed by 20 strokes with pestle B (tight). The grinding and filtering parameters were adjusted, depending on the tissue type. Considering the possibility that density gradient centrifugation would specifically enrich certain types of nuclei, we skipped this step to avoid any sampling bias. After tissue grinding, nuclei suspensions were collected and centrifuged for $5 \mathrm{~min}$ at $500 \times \mathrm{g}$ in a $4^{\circ} \mathrm{C}$ prechilled swinging-bucket centrifuge. After removing the supernatant, nuclei pellets were washed twice with chilled wash buffer (10 mM Tris- $\mathrm{HCl} \mathrm{pH}$ 8.0, $25 \mathrm{mM} \mathrm{KCl,} 5 \mathrm{mM} \mathrm{MgCl}_{2}$, $250 \mathrm{mM}$ sucrose, $0.1 \%$ Tween-20, $1 \times$ protease inhibitor cocktail, and $0.1 \mathrm{mM}$ DTT in nuclease-free water). Then nuclei were counted by DAPI (Beyotime, C1006) staining.

\section{ATAC-Seq Library Construction}

For the transposition step, we used 50,000 nuclei per each reaction. The transposition reaction mix contains $10 \mathrm{mM}$ TAPS$\mathrm{NaOH}$ (pH 8.5), $5 \mathrm{mM} \mathrm{MgCl}, 10 \%$ DMF, $2.5 \mu \mathrm{L}$ of in-house Tn5 transposase $(0.8 \mathrm{U} / \mu \mathrm{L})$ and phosphate-buffered saline (Gibco, 10010-031) (Buenrostro et al., 2015; Liu et al., 2019). Those reactions were incubated at $37^{\circ} \mathrm{C}$ for $30 \mathrm{~min}$ in a thermomixer shaking at 500 RPM. After transposition, the transposed DNA was purified with Qiagen MinElute PCR Purification Kit (Qiagen, 28006), and purified products were amplified with barcoded primers, NEBnext High-Fidelity PCR master mix (NEB, M0541S) as previously described. Libraries were quality controlled using Agilent High Sensitivity Assay (Agilent, 5067-4626). The library construction was considered successful upon confirmation of the correct average fragment size.

\section{Sequencing}

All sequencing data were generated from MGISEQ-2000 platform (MGI). After size selection, libraries were quantified by Qubit dsDNA HS Assay Kit 3.0 (Invitrogen, Q32854). The polymerase chain reaction product from eight pooled libraries was heat-denatured and ligated into single-strand circular DNA. After DNB generation, libraries were then loaded on sequencing chip and sequenced with paired-end 50-bp reads (Huang et al., 2017).

\section{Preprocessing of the ATAC-Seq Datasets}

After configuring the rat genome (rn6) and annotation files, the ATAC-seq raw data were processed by trimming, aligning, filtering, and quality controlling following an ATAC-seq pipeline from Kundaje lab (Koh et al., 2016). We used MACS2 (Zhang et al., 2008) (version 2.1.2) to identify peaks with options -B, -q 0.01 , -nomodel, -f BAM. To evaluate the reproducibility between biological replicates of each tissue, we used the Irreproducible Discovery Rate method (Li et al., 2011) to identify overlapping peaks between replicates. Only the peaks present in both biological replicates were retained for subsequent analysis. We established the standard peak by merging overlapping peaks from all tissues, and we calculated the distance from nearest transcription start site (TSS) by using distance ToNearest function in GenomicRanges package (Lawrence et al., 2013). Then, we obtained the raw count matrix by using BedTools (Quinlan and Hall, 2010) (version 2.26.0) intersect function to count the reads mapped to each standard peak. We normalized the raw count matrix by reads per million mapped reads (RPM) and calculated 
Pearson correlation coefficients based on the $\log _{10}$ transformed RPM matrix.

\section{Identification of Tissue-Specific Chromatin Accessible Regions}

Previously described strategy based on Shannon entropy (Schug et al., 2005; Barrera et al., 2008; Shen et al., 2012) was used to compute tissue-specific index. We defined the relative accessibility of each peak in a tissue type i as $R i=E i / \Sigma E$, where $E i$ is the RPM value of the peak in the tissue $i, \Sigma E$ is the sum of RPM values in all tissues, and $N$ is the total number of tissues. For each peak, the entropy score across tissues can be defined as $H=-1{ }^{*} \operatorname{sum}\left(R i^{*} \log _{2} R i\right)(1<i<N)$, where the value of $H$ ranges between 0 and $\log _{2}(N)$. A highly tissue-specific peak owned an entropy score close to zero, while if a peak is conserved between different tissues, its entropy score close to $\log _{2}(N)$ (Xie et al., 2013). Based on the distribution of entropy scores in our datasets, peaks with score $<3$ were identified as body organspecific peaks, and peaks with score $<2$ were identified as brain region-specific peaks.

We searched TF motifs with the findMotifsGenome.pl script of the HOMER (Heinz et al., 2010) version 4.9.1 software and generated a motif enrichment matrix, where each row represents the $P$-value of a motif, and each column represents a tissue. We displayed the top 15 motifs of each tissue.

\section{RESULTS}

\section{ATAC-Seq Data Quality Control}

A total of 72 frozen samples from 32 tissues were collected from two female and two male rats and used for bulk ATACseq profiling (Figure 1A). In total, we obtained an average of 77 million reads per sample and identified 34,219 body organ-specific and 38,502 brain-specific chromatin accessible elements from a total of 397,691 peaks (Supplementary Table 2). First, we systematically evaluated the quality of ATAC-seq datasets on several parameters including total raw read number, clean read number, mapping rate, and peak number (Supplementary Table 2). The enrichment score of reads at the TSS indicated our ATAC-seq datasets to be of high quality (Figure 1B). By calculating the proportions of ATACseq peaks annotated to different genomic regions, we observed the largest proportion of ATAC-seq peaks was annotated to distal peaks (Figure 1C), in agreement with the knowledge of distal regulatory elements being more abundant and important in ATAC-seq data (Buenrostro et al., 2013). Second, we evaluated the correlations between biological replicates obtained from either the same or opposite gender with Pearson correlation coefficients and observed high correlation. Specifically, the correlation between those of the same gender was subtly higher than that of the opposite gender (Figure 1D). As expected, heatmap clustering showed that tissue samples from the same organ were highly correlated, further demonstrating the reproducibility between biological replicates (Figure 1E). Furthermore, our analysis showed high correlations among five distinct cerebral cortex regions (motor, prefrontal, auditory, primary visual, and somatosensory cortex), whereas it showed lower correlations between brain regions of cerebellum, olfactory bulb, cerebral cortex, hippocampus, and other tissue types. In summary, our data survey reveals our rat ATAC-seq dataset to be of high quality and thus can be used to reliably detect chromatin accessible regions throughout the rat genome.

\section{Identification of Tissue-Specific Chromatin Accessible Peaks and Transcription Factors}

We next identified all genome-wide tissue-specific chromatin accessible regions as illustrated in Figures 2A,B. For tissuespecific genes, we provide integrative genomics viewer results showing higher ATAC-seq enrichment at annotated or putative promoters and enhancers (Figure 2C and Supplementary Figure 1A). For example, in spleen, ATACseq peaks are enriched around $M s 4 a 1$, which is a specific membrane protein gene expressed in B lymphocytes (Zuccolo et al., 2013) (Figure 2C). Similarly, analysis of the olfactory bulb revealed the enrichment of peaks near the Cpa6 promoter, which is consistent with previous finding that $C p a 6$ is highly expressed in the mitral and granular cell layers of olfactory bulb of adult mice as revealed by in situ hybridization (Fontenele-Neto et al., 2005) (Supplementary Figure 1A).

To verify the putative tissue-specific regulatory regions identified in our rat ATAC-seq data, we systematically compared those with existing studies from other species. By doing so, we found that tissue-specific transcription factors identified in rats are also highly consistent with those in other species. For example, previous studies have shown that HNF1b is an important transcriptional regulator of renal epithelial tissue (Ferrè and Igarashi, 2019), whereas HNF4a and HNF6a are key regulators during cellular differentiation in liver (Nagaki and Moriwaki, 2008). Our rat ATAC-seq atlas further confirmed that motifs of HNF1, HNF1b, HNF4a, HNF6, and HNF (hepatocyte nuclear factor) family transcription factors are enriched in liver, pancreas, ileum, and kidney in both rat and mice (Figure 2D). In addition, it has been reported that RUNX1 interplays with FOXL2 to maintain the identity of fetal ovary and secure the identity of ovarian-supporting cells (Nicol et al., 2019). Consistent with this, we found that RUNX1 motif is highly specific in rat ovary (Figure 2D). Furthermore, the MEF2 (myocyte enhancer factor 2, including MEF2a, MEF2b, MEF2c, MEF2d) family of transcription factors is a key regulator of cardiac muscle differentiation and development, as shown by the knockout of Mef2a gene, leading to dramatic changes of gene expression in the heart chamber (Medrano and Naya, 2017). MEF2a also plays a role in neuronal survival involved in memory and learning (Dietrich, 2013). Consistently, we also validated the enrichment of MEF2a in both heart and central neuron system in our rat atlas (Figure 2D and Supplementary Figure 1B).

In order to better understand the relationship between brain regions and social play behaviors, we also analyzed brain datasets to identify region-specific transcription factors. We first merged the datasets across different cerebral cortex for downstream region-specific peak analysis, as we did not observe significant differences in enriched transcription factors within cerebral cortex areas. A collection of brain region-specific peaks was identified among amygdala, cerebellum, cerebral cortex, hippocampus, and other regions (Figure 2B). In line 
A

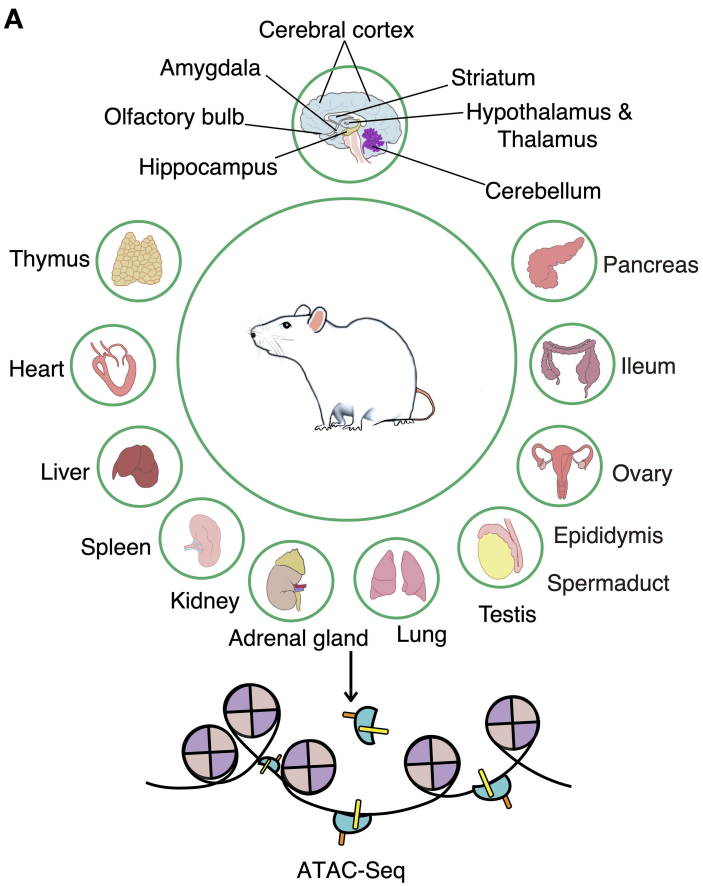

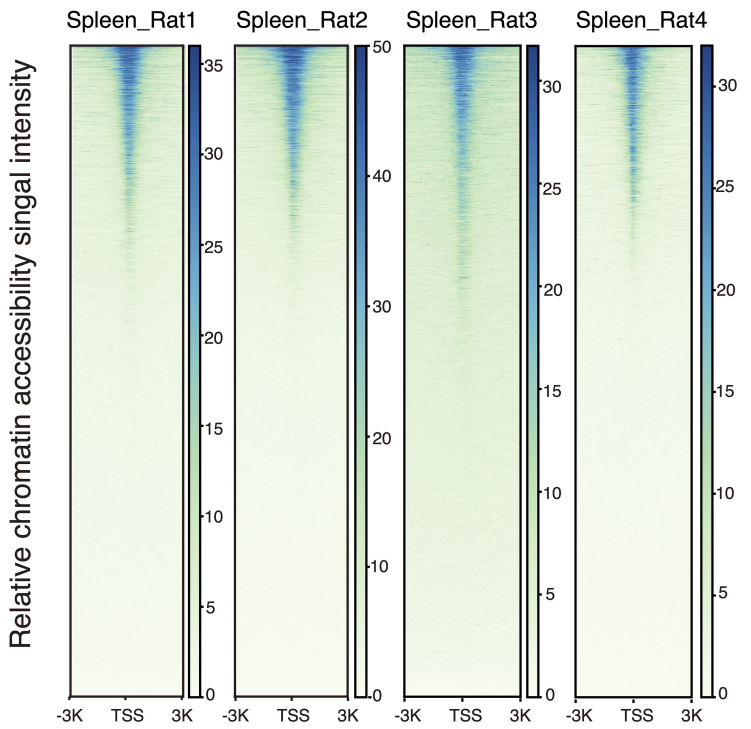

C

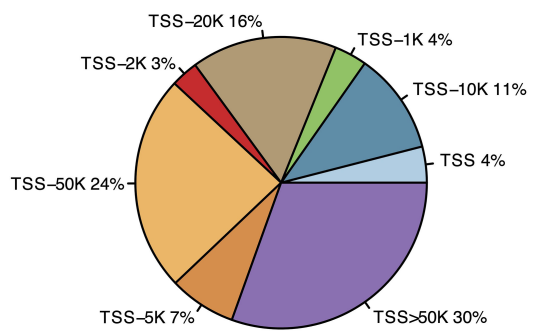

D
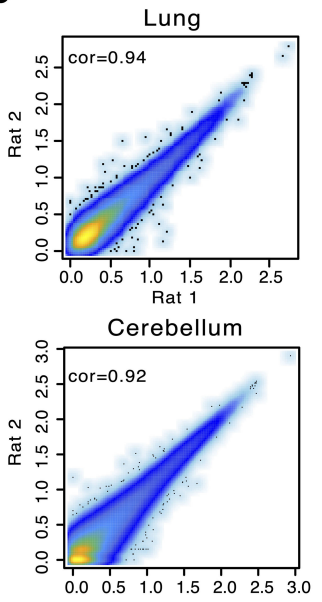

Rat 1

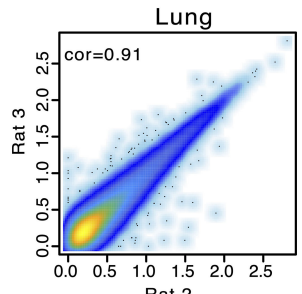

Rat 2

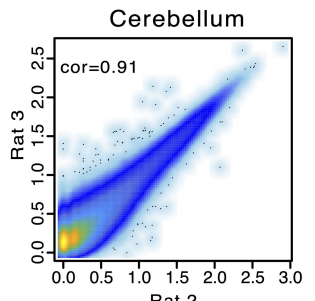

E

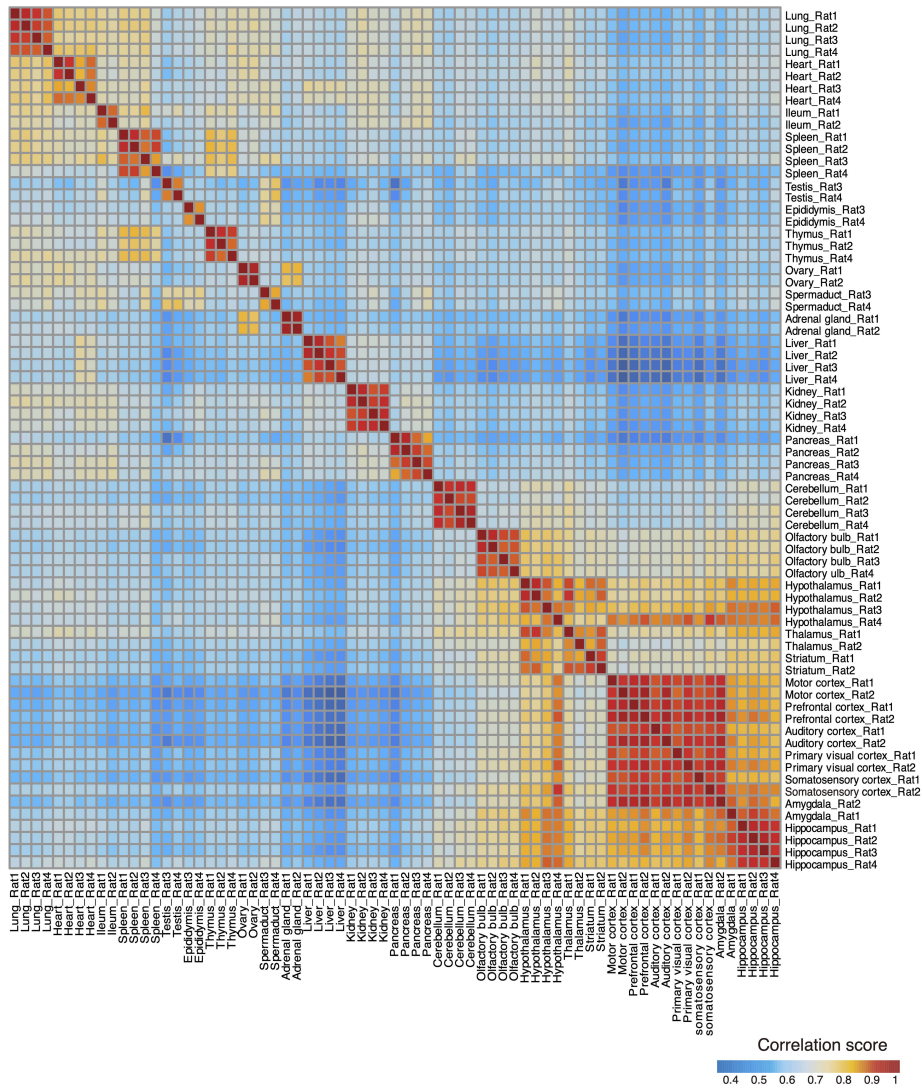

FIGURE 1 | ATAC-seq data quality control and evaluation of reproducibility. (A) Thirty-six different tissues from adult female and male rats were collected for ATAC-seq profiling. (B) The ATAC-seq signal enrichment around TSSs for spleen samples (Rat1 and Rat2 represent female rats; Rat3 and Rat4 represent male rats).

(C) Proportions of ATAC-seq peaks annotated to different genomic regions. (D) Scatter plots showing the Pearson correlations between biological replicates for two representative samples (lung and cerebellum of female and male rats). (E) Heatmap clustering across all 71 tissue ATAC-seq profiles. 
A

Body organs specific peaks

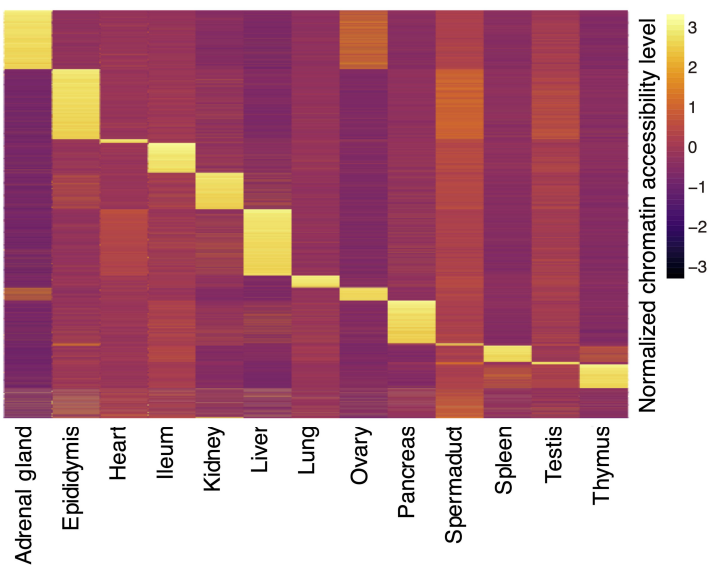

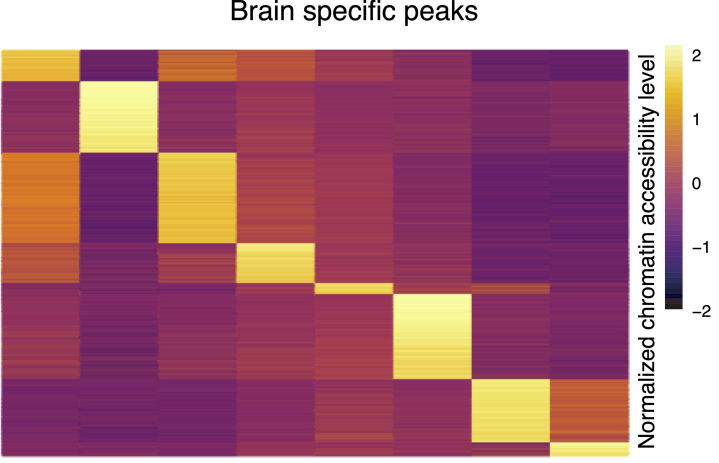

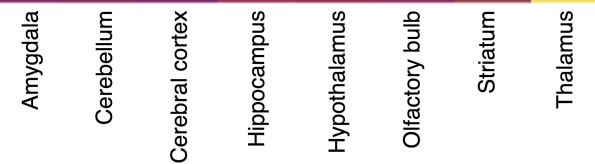

C

D

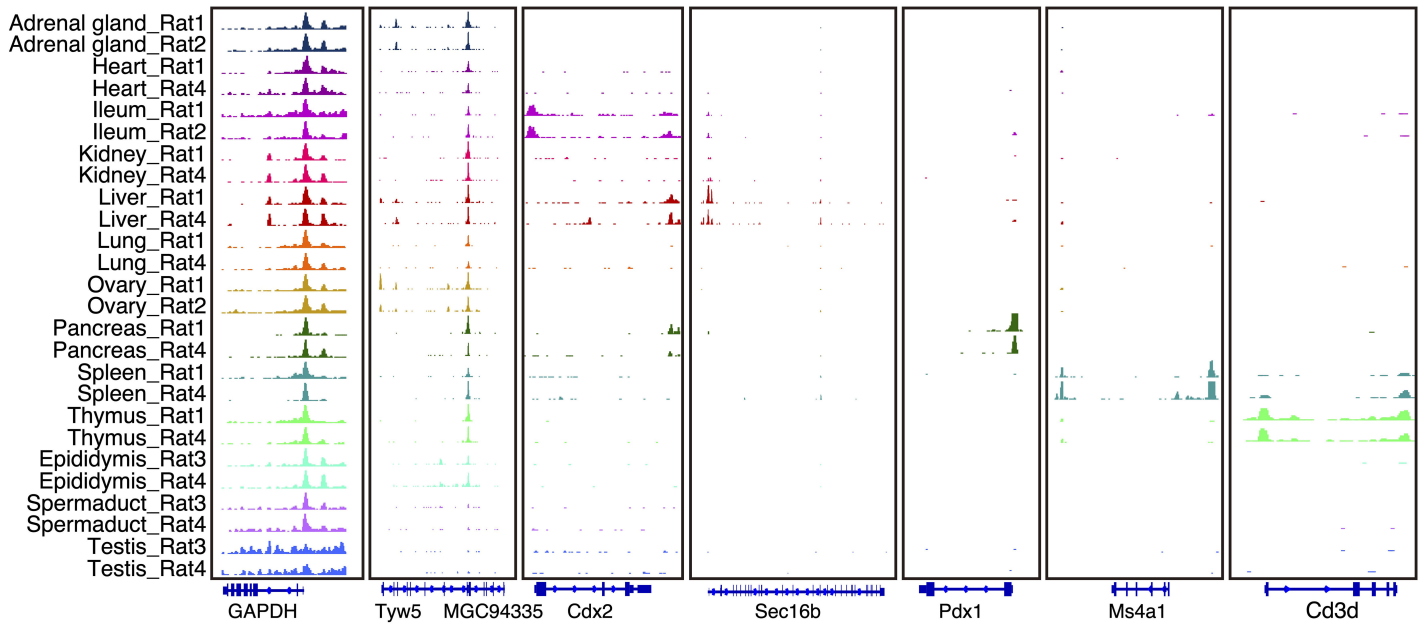

Transcription factor

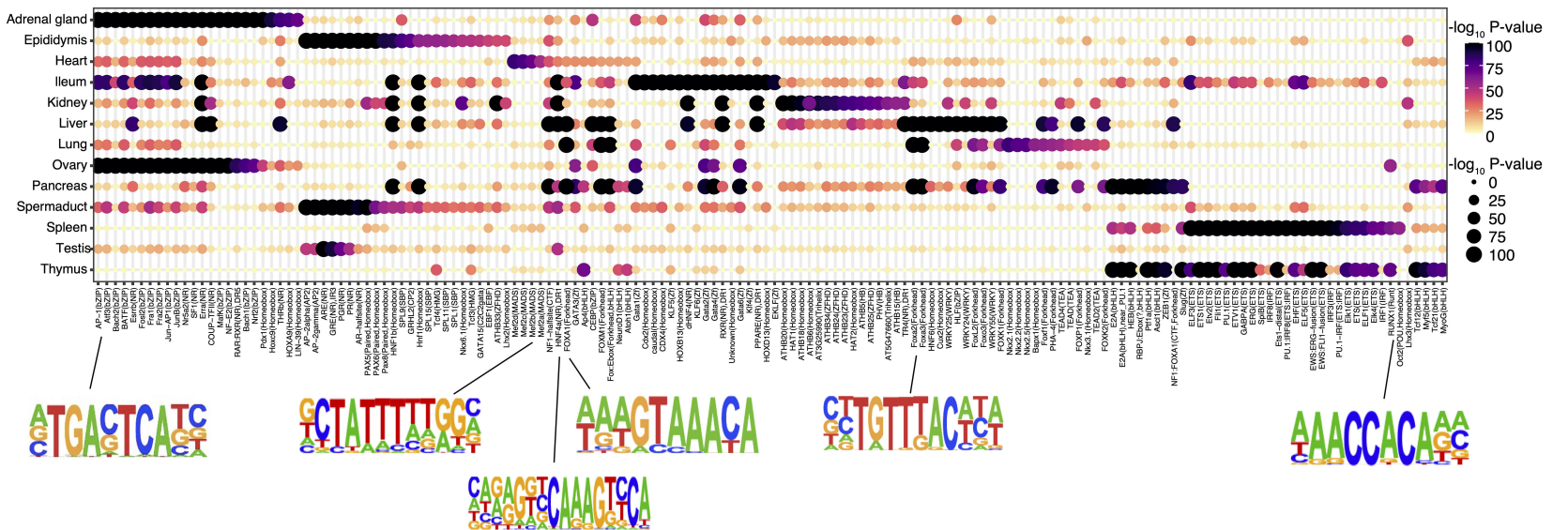

FIGURE 2 | The landscape of tissue-specific chromatin accessibility and transcription factors. (A) Heatmap clustering showing the body organ-specific accessible elements. (B) Heatmap clustering showing the brain-specific accessible elements. (C) The integrative genomics viewer shows enrichment of ATAC-seq signal for the indicated housekeeping gene (Gapdh) and body organ-specific genes. (D) Enrichment of the indicated TF motifs in each tissue. The size and color of each point represent the motif enrichment $P$-value $\left(-\log _{10} P\right.$-value). 
with previous studies, we observed significant enrichments of NEUROG2, ATOH1, OLIG2, and NEUROD1 transcription factors in amygdala, cerebellum, cerebral cortex, and hippocampus (Supplementary Figure 1B). These transcription factors belong to the basic-helix-loop-helix (bHLH) family, and their function is crucial to determine the fate and differentiation of neural cells to ensure that different brain regions are supplied with the appropriate number of neuronal and glial cells (Dennis et al., 2019). Furthermore, transcription factor EGR1 has been shown to be related to the long-term fear memory and anxiety (Ko et al., 2005); our brain datasets unbiasedly identified EGR1 enrichment in the amygdala and hippocampus (the main brain areas for memory formation and storage), thus further validated previous findings.

\section{CONCLUSIONS}

In summary, we have profiled the chromatin accessibility using ATAC-seq for 10 body tissues and 12 brain regions from adult rats and produced a large dataset with replicates. This comprehensive chromatin accessibility atlas contains 397,691 accessible elements. In addition, by comparing the open chromatin landscapes among rat tissues, a total of 34,219 body organ-specific peaks, 38,502 brain-specific peaks and a list of putative tissue-specific transcription factors were unbiasedly identified. We further showed that many known tissue-specific transcriptional characteristics can be recapitulated in this study, indicating that our data resource is of high quality and will be useful for future mechanistic discoveries in diseases and social behaviors.

\section{DATA AVAILABILITY STATEMENT}

All raw data have been submitted to the CNGB [Nucleotide Sequence Archive] [https://db.cngb.org/search/project/ CNP0001470/] and also the NCBI [SRA] [https://www. ncbi.nlm.nih.gov/bioproject/PRJNA684678]. The ATAC-seq QC results have been submitted to Figshare under https://doi.org/10. 6084/m9.figshare.13370498.v6.

\section{ETHICS STATEMENT}

The animal study was reviewed and approved by Institutional Review Board on Ethics Committee of BGI.

\section{REFERENCES}

Abbott, A. (2009). Return of the rat. Nature 460:788. doi: 10.1038/460788a

Adams, M. D., Celniker, S. E., Holt, R. A., Evans, C. A., Gocayne, J. D., Amanatides, P. G., et al. (2000). The genome sequence of Drosophila melanogaster. Science 287, 2185-2195. doi: 10.1126/science.287. 5461.2185

Alugubelly, N., Mohammad, A. N., Edelmann, M. J., Nanduri, B., Sayed, M., Park, J. W., et al. (2019). Proteomic and transcriptional profiling of rat amygdala following social play. Behav. Brain Res. 376:112210. doi: 10.1016/j.bbr.2019.112210

Baldini, A. (2010). Mouse as a Model for Human Disease. Vogel and Motulsky's Human Genetics. Berlin; Heidelberg: Springer, 779-785. doi: 10.1007/978-3-540-37654-5_34

\section{AUTHOR CONTRIBUTIONS}

YYua, QD, XW, CL, and LL conceived the idea. YJ, PG, and XZ collected samples. YYua, QD, and YYu generated the data. JX, PW, MC, and LH assisted with the experiments. XW analyzed the data with the assistance of YYua and YLi. YYua, wrote the manuscript with the input of XW. CL and LL supervised the study. LL, QL, GV, and CY revised the manuscript. YLa, ME, and $\mathrm{HY}$ provided helpful comments on this study. All authors reviewed and approved the final manuscript.

\section{FUNDING}

This work was supported by the Guangdong Provincial Academician Workstation of BGI Synthetic Genomics (No. 2017B090904014). ME was supported by the Strategic Priority Research Program of the Chinese Academy of Sciences (No. XDA16030502) and the Innovative Team Program of Guangzhou Regenerative Medicine and Health Guangdong Laboratory (No. 2018GZR110103001).

\section{ACKNOWLEDGMENTS}

We thank all members of the Center for Digitizing Cells from Institute of SuperCells (BGI) for helpful comments. We thank China National GeneBank for providing sequencing service for this project.

\section{SUPPLEMENTARY MATERIAL}

The Supplementary Material for this article can be found online at: https://www.frontiersin.org/articles/10.3389/fgene. 2021.651604/full\#supplementary-material

Supplementary Figure 1 | The landscape of brain-specific chromatin accessibility and transcription factors. (A) The integrative genomics viewer shows enrichment of ATAC-seq signal for the indicated housekeeping gene (Gapdh) and brain-specific genes. (B) Enrichment of the indicated TF motifs in each tissue. The size and color of each point represent the motif enrichment $P$-value $\left(-\log _{10}\right.$ $P$-value).

Supplementary Table 1 | Tissue and corresponding rat and sample IDs.

Supplementary Table 2 | ATAC-seq metadata and mapping statistics.

Barrera, L. O., Li, Z., Smith, A. D., Arden, K. C., Cavenee, W. K., Zhang, M. Q., et al. (2008). Genome-wide mapping and analysis of active promoters in mouse embryonic stem cells and adult organs. Genome Res. 18, 46-59. doi: $10.1101 /$ gr.6654808

Buenrostro, J. D., Giresi, P. G., Zaba, L. C., Chang, H. Y., and Greenleaf, W. J. (2013). Transposition of native chromatin for fast and sensitive epigenomic profiling of open chromatin, DNA-binding proteins and nucleosome position. Nat. Methods 10, 1213-1218. doi: 10.1038/nmeth.2688

Buenrostro, J. D., Wu, B., Chang, H. Y., and Greenleaf, W. J. (2015). ATAC-seq: a method for assaying chromatin accessibility genome-wide. Curr. Protoc. Mol. Biol. 109, 21-29. doi: 10.1002/0471142727.mb2129s109

Cao, J., O’day, D. R., Pliner, H. A., Kingsley, P. D., Deng, M., Daza, R. M., et al. (2020). A human cell atlas of fetal gene expression. Science. 370:eaba7721. doi: 10.1126/science.aba7721 
Collins, F. S., Morgan, M., and Patrinos, A. (2003). The human genome project: lessons from large-scale biology. Science 300, 286-290. doi: 10.1126/science.10 84564

Corces, M. R., Trevino, A. E., Hamilton, E. G., Greenside, P. G., SinnottArmstrong, N. A., Vesuna, S., et al. (2017). An improved ATAC-seq protocol reduces background and enables interrogation of frozen tissues. Nat. Methods 14, 959-962. doi: 10.1038/nmeth.4396

Cusanovich, D. A., Hill, A. J., Aghamirzaie, D., Daza, R. M., Pliner, H. A., Berletch, J. B., et al. (2018a). A single-cell atlas of in vivo mammalian chromatin accessibility. Cell 174, 1309-1324 e1318. doi: 10.1016/j.cell.2018.06.052

Cusanovich, D. A., Reddington, J. P., Garfield, D. A., Daza, R. M., Aghamirzaie, D., Marco-Ferreres, R., et al. (2018b). The cis-regulatory dynamics of embryonic development at single-cell resolution. Nature 555, 538-542. doi: 10.1038/nature25981

Dahl, J. A., Jung, I., Aanes, H., Greggains, G. D., Manaf, A., Lerdrup, M., et al. (2016). Broad histone H3K4me3 domains in mouse oocytes modulate maternal-to-zygotic transition. Nature 537, 548-552. doi: 10.1038/nature19360

Daugherty, A. C., Yeo, R. W., Buenrostro, J. D., Greenleaf, W. J., Kundaje, A., and Brunet, A. (2017). Chromatin accessibility dynamics reveal novel functional enhancers in C. elegans. Genome Res. 27, 2096-2107. doi: $10.1101 /$ gr.226233.117

Dennis, D. J., Han, S., and Schuurmans, C. (2019). bHLH transcription factors in neural development, disease, and reprogramming. Brain Res. 1705, 48-65. doi: 10.1016/j.brainres.2018.03.013

Dietrich, J.-B. (2013). The MEF2 family and the brain: from molecules to memory. Cell Tissue Res. 352, 179-190. doi: 10.1007/s00441-013-1565-2

Domcke, S., Hill, A. J., Daza, R. M., Cao, J., O'day, D. R., Pliner, H. A., et al. (2020). A human cell atlas of fetal chromatin accessibility. Science. 370:eaba7612. doi: $10.1126 /$ science.aba7612

Ferrè, S., and Igarashi, P. (2019). New insights into the role of HNF$1 \mathrm{~b}$ in kidney (patho) physiology. Pediatric Nephrol. 34, 1325-1335. doi: 10.1007/s00467-018-3990-7

Fontenele-Neto, J. D., Kalinina, E., Feng, Y., and Fricker, L. D. (2005). Identification and distribution of mouse carboxypeptidase A-6. Mol. Brain Res. 137, 132-142. doi: 10.1016/j.molbrainres.2005.02.026

Gerstein, M. B., Lu, Z. J., Van Nostrand, E. L., Cheng, C., Arshinoff, B. I., Liu, T., et al. (2010). Integrative analysis of the Caenorhabditis elegans genome by the modENCODE project. Science 330, 1775-1787. doi: 10.1126/science.1196914

Gibbs, R. A., and Pachter, L. (2004). Genome sequence of the Brown Norway rat yields insights into mammalian evolution. Nature 428, 493-521. doi: 10.1038 /nature02426

Graveley, B. R., Brooks, A. N., Carlson, J. W., Duff, M. O., Landolin, J. M., Yang, L., et al. (2011). The developmental transcriptome of Drosophila melanogaster. Nature 471, 473-479. doi: 10.1038/nature09715

Gurumurthy, C. B., Quadros, R. M., Richardson, G. P., Poluektova, L. Y.,Mansour, S. L., and Ohtsuka, M. (2020). Genetically modified mouse models to help fight COVID-19. Nat. Protocols. 15, 3777-3787. doi: 10.1038/s41596-020-00403-2

Han, X., Wang, R., Zhou, Y., Fei, L., Sun, H., Lai, S., et al. (2018). Mapping the mouse cell atlas by microwell-seq. Cell 172, 1091-1107. e1017. doi: 10.1016/j.cell.2018.02.001

Heinz, S., Benner, C., Spann, N., Bertolino, E., Lin, Y. C., Laslo, P., et al. (2010). Simple combinations of lineage-determining transcription factors prime cis-regulatory elements required for macrophage and B cell identities. Mol. Cell 38, 576-589. doi: 10.1016/j.molcel.2010. 05.004

Huang, J., Liang, X., Xuan, Y., Geng, C., Li, Y., Lu, H., et al. (2017). A reference human genome dataset of the BGISEQ-500 sequencer. Gigascience 6:gix024. doi: 10.1093/gigascience/gix024

Jacob, H. J. (2010). The rat: a model used in biomedical research. Methods Mol. Biol. 597:1. doi: 10.1007/978-1-60327-389-3_1

Ko, S. W., Hu-Shan, A., Gallitano-Mendel, A., Chang-Shen, Q., Feng, W., Milbrandt, J., et al. (2005). Transcription factor Egr-1is required for long-term fear memory and anxiety. Acta Physiol. Sin. 57, 421-432.

Koh, P. W., Sinha, R., Barkal, A. A., Morganti, R. M., Chen, A., Weissman, I. L., et al. (2016). An atlas of transcriptional, chromatin accessibility, and surface marker changes in human mesoderm development. Sci. Data 3, 1-15. doi: 10.1038/sdata.2016.109

Lawrence, M., Huber, W., Pages, H., Aboyoun, P., Carlson, M., Gentleman, R., et al. (2013). Software for computing and annotating genomic ranges. PLoS Comput. Biol. 9:e1003118. doi: 10.1371/journal.pcbi.1003118
Li, J. J., Huang, H., Bickel, P. J., and Brenner, S. E. (2014). Comparison of D. melanogaster and C. elegans developmental stages, tissues, and cells by modENCODE RNA-seq data. Genome Res. 24, 1086-1101. doi: 10.1101/gr.170100.113

Li, Q., Brown, J. B., Huang, H., and Bickel, P. J. (2011). Measuring reproducibility of high-throughput experiments. Ann. Appl. Stat. 5, 1752-1779. doi: 10.1214/11-AOAS466

Liu, C., Wang, M., Wei, X., Wu, L., Xu, J., Dai, X., et al. (2019). An ATACseq atlas of chromatin accessibility in mouse tissues. Sci. Data 6, 1-10. doi: 10.1038/s41597-019-0071-0

Liu, X., Wang, C., Liu, W., Li, J., Li, C., Kou, X., et al. (2016). Distinct features of $\mathrm{H} 3 \mathrm{~K} 4 \mathrm{me} 3$ and $\mathrm{H} 3 \mathrm{~K} 27 \mathrm{me} 3$ chromatin domains in pre-implantation embryos. Nature 537, 558-562. doi: 10.1038/nature19362

Meaney, M. J., and Stewart, J. (1981). A descriptive study of social development in the rat (Rattus norvegicus). Anim. Behav. 29, 34-45. doi: 10.1016/S0003-3472(81)80149-2

Medrano, J. L., and Naya, F. J. (2017). The transcription factor MEF2A fine-tunes gene expression in the atrial and ventricular chambers of the adult heart. J. Biol. Chem. 292, 20975-20988. doi: 10.1074/jbc.M117.806422

Nagaki, M., and Moriwaki, H. (2008). Transcription factor HNF and hepatocyte differentiation. Hepatol. Res. 38, 961-969. doi: 10.1111/j.1872-034X.2008.00367.x

Nicol, B., Grimm, S. A., Chalmel, F., Lecluze, E., Pannetier, M., Pailhoux, E., et al. (2019). RUNX1 maintains the identity of the fetal ovary through an interplay with FOXL2. Nat. Commun. 10, 1-14. doi: 10.1038/s41467-019-13060-1

Park, J., Shrestha, R., Qiu, C., Kondo, A., Huang, S., Werth, M., et al. (2018). Singlecell transcriptomics of the mouse kidney reveals potential cellular targets of kidney disease. Science 360, 758-763. doi: 10.1126/science.aar2131

Quinlan, A. R., and Hall, I. M. (2010). BEDTools: a flexible suite of utilities for comparing genomic features. Bioinformatics 26, 841-842. doi: 10.1093/bioinformatics/btq033

Schug, J., Schuller, W.-P., Kappen, C., Salbaum, J. M., Bucan, M., and Stoeckert, C. J. (2005). Promoter features related to tissue specificity as measured by Shannon entropy. Genome Biol. 6:R33. doi: 10.1186/gb-2005-6-4-r33

Schweinfurth, M. K. (2020). The social life of Norway rats (Rattus norvegicus). Elife 9:e54020. doi: 10.7554/eLife.54020

Shen, Y., Yue, F., Mccleary, D. F., Ye, Z., Edsall, L., Kuan, S., et al. (2012). A map of the cis-regulatory sequences in the mouse genome. Nature 488, 116-120. doi: $10.1038 /$ nature 11243

Söllner, J. F., Leparc, G., Hildebrandt, T., Klein, H., Thomas, L., Stupka, E., et al. (2017). An RNA-Seq atlas of gene expression in mouse and rat normal tissues. Sci. Data 4:170185. doi: 10.1038/sdata.2017.185

Wu, J., Huang, B., Chen, H., Yin, Q., Liu, Y., Xiang, Y., et al. (2016). The landscape of accessible chromatin in mammalian preimplantation embryos. Nature 534, 652-657. doi: 10.1038/nature18606

Xie, W., Schultz, M. D., Lister, R., Hou, Z., Rajagopal, N., Ray, P., et al. (2013). Epigenomic analysis of multilineage differentiation of human embryonic stem cells. Cell 153, 1134-1148. doi: 10.1016/j.cell.2013.04.022

Zhang, B., Zheng, H., Huang, B., Li, W., Xiang, Y., Peng, X., et al. (2016). Allelic reprogramming of the histone modification $\mathrm{H} 3 \mathrm{~K} 4 \mathrm{me} 3$ in early mammalian development. Nature 537, 553-557. doi: 10.1038/nature19361

Zhang, Y., Liu, T., Meyer, C. A., Eeckhoute, J., Johnson, D. S., Bernstein, B. E., et al. (2008). Model-based analysis of ChIP-Seq (MACS). Genome Biol. 9, 1-9. doi: 10.1186/gb-2008-9-9-r137

Zuccolo, J., Deng, L., Unruh, T., Sanyal, R., Bau, J. A., Storek, J., et al. (2013). Expression of MS4A and TMEM176 genes in human B lymphocytes. Front. Immunol. 4:195. doi: 10.3389/fimmu.2013.00195

Conflict of Interest: The authors declare that the research was conducted in the absence of any commercial or financial relationships that could be construed as a potential conflict of interest.

Copyright (C) 2021 Yuan, Deng, Wei, Liu, Lan, Jiang, Yu, Guo, Xu, Yu, Han, Cheng, Wu, Zhang, Lai, Volpe, Esteban, Yang, Liu and Liu. This is an open-access article distributed under the terms of the Creative Commons Attribution License (CC BY). The use, distribution or reproduction in other forums is permitted, provided the original author(s) and the copyright owner(s) are credited and that the original publication in this journal is cited, in accordance with accepted academic practice. No use, distribution or reproduction is permitted which does not comply with these terms. 